PROCEEDINGS OF THE

AMERICAN MATHEMATICAL SOCIETY

Volume 132, Number 1, Pages 157-164

S 0002-9939(03)07039-4

Article electronically published on May 8, 2003

\title{
ON THE SECOND EIGENVALUE OF THE LAPLACE OPERATOR ON A SPHERICAL BAND
}

\author{
CHUNG-TSUN SHIEH \\ (Communicated by Carmen C. Chicone)
}

\begin{abstract}
In this paper we prove that the second eigenvalue of the Laplacian for a spherical band on the unit sphere $S^{2}$ has multiplicity 2. We also show that among all spherical bands of given fixed area less than $2 \pi$ the second eigenvalue is maximized at the band which is symmetrical with respect to the equator.
\end{abstract}

\section{INTRODUCTION}

On the unit sphere $S^{2}$, if we introduce the Euler coordinate

$$
\begin{array}{ll}
x=\sin \phi \cos \theta, & y=\sin \phi \sin \theta, \quad z=\cos \phi, \\
0 \leq \phi \leq \pi, & 0 \leq \theta<2 \pi,
\end{array}
$$

then the Laplace operator $\Delta_{S^{2}}$ on $S^{2}$ can be written as

$$
\Delta_{S^{2}} u(\phi, \theta)=\frac{1}{\sin \phi}\left[\frac{\partial}{\partial \phi}\left(\sin \phi \frac{\partial u}{\partial \phi}\right)+\frac{\partial}{\partial \theta}\left(\frac{1}{\sin \phi} \frac{\partial u}{\partial \theta}\right)\right](\theta, \phi) .
$$

For $0<\phi_{1}<\phi_{2} \leq \pi$, the set

$$
\mathcal{B}\left(\phi_{1}, \phi_{2}\right)=\left\{(x, y, z) \in S^{2}: \phi_{1} \leq \phi \leq \phi_{2}\right\}
$$

shall be called a spherical band in $S^{2}$. For $\mathcal{B}=\mathcal{B}\left(\phi_{1}, \phi_{2}\right)$, consider the following problem:

$$
\Delta_{S^{2}} u+\lambda u=0, \quad u=0 \text { on } \partial \mathcal{B} .
$$

Let $\left\{\lambda_{n}(\mathcal{B})\right\}_{n=1}^{\infty}$ denote the set of eigenvalues of (1.2), which are arranged in ascending order as

$$
\lambda_{1}(\mathcal{B})<\lambda_{2}(\mathcal{B}) \leq \cdots
$$

The multiplicity of $\lambda_{n}(\mathcal{B})$ is the dimension of the space of eigenfunctions of (1.2) corresponding to $\lambda_{n}(\mathcal{B})$. In [7] we proved that, among all bands $\mathcal{B}$ of given area, $\lambda_{1}(\mathcal{B})$ attains its maximum when $\mathcal{B}$ is symmetric with respect to the equator. More precisely, we proved that $\lambda_{1}(\mathcal{B})$ is decreasing as $\mathcal{B}$ moves from the equatorially symmetric band towards a pole. In this paper we prove that the multiplicity of $\lambda_{2}(\mathcal{B})$ is two for any band, and that among all bands of given fixed area smaller than $2 \pi$ the function $\lambda_{2}(\mathcal{B})$ attains its maximum at the band which is symmetric with respect to the equator.

Received by the editors April 27, 2001 and, in revised form, July 8, 2002 and August 21, 2002. 2000 Mathematics Subject Classification. Primary 35P15.

(C)2003 American Mathematical Society 


\section{The multiplicity of the SECOnd eigenvalue OF A SPHERICAL BAND}

Employing the technique of separation of variables in (1.2) by letting $u(\phi, \theta)=$ $v(\phi) w(\theta)$ we separate (1.2) into two equations,

$$
\begin{gathered}
v^{\prime \prime}(\phi)+\cot \phi v^{\prime}(\phi)+\left(\lambda-\frac{c^{2}}{\sin ^{2} \phi}\right) v(\phi)=0, v\left(\phi_{1}\right)=v\left(\phi_{2}\right)=0, \\
w^{\prime \prime}(\theta)+c^{2} w(\theta)=0, w^{(j)}(0)=w^{(j)}(2 \pi)=0, j=0,1 .
\end{gathered}
$$

Note that the eigenvalues of the periodic eigenvalue problem (2.2) are of the form $c_{n}^{2}=n^{2}, n=0,1, \cdots ; 0$ is a simple eigenvalue of $(2.2)$, for $n>0$ the multiplicity of $n^{2}$ is two. If we denote the $k$ th eigenvalue and the corresponding eigenfunction of the Dirichlet eigenvalue of (2.1) by $\lambda_{k}(c)$ and $v_{k}(\phi ; c)$, respectively, then the spectrum of (1.1) consists of $\lambda_{k}(n)$, where $k=1,2, \cdots$ and $n=0,1,2, \cdots$. Note that $\lambda_{1}=\lambda_{1}(0)$, which is a simple eigenvalue, and the eigenfunctions corresponding to $\lambda_{k}(n)$ are linear combinations of $v_{k}(\phi ; n) \cos n \theta$ and $v_{k}(\phi ; n) \sin n \theta$. To determine the multiplicity of an eigenvalue $\lambda_{*}$ of (1.1) it is sufficient to find how many $k$ 's and $n$ 's are such that $\lambda_{k}(n)=\lambda_{*}$. This problem is in general difficult to deal with, but for the second eigenvalue of (1.1) the problem is much simpler. Indeed, Courant's nodal domain theorem tells us that the second eigenfunction of (1.1) has two nodal domains. On the other hand, among the eigenfunctions of (1.1) constructed above, only $v_{2}(\phi ; 0), v_{1}(\phi ; 1) \cos \theta$ and $v_{1}(\phi ; 1) \sin \theta$ have two nodal domains. Thus if we know the smallest between $\lambda_{2}(0)$ and $\lambda_{1}(1)$, then we can determine the multiplicity of $\lambda_{2}$. The following theorem answers this question.

Theorem 2.1. $\lambda_{2}(\mathcal{B})=\lambda_{1}(1)$. The multiplicity of the second eigenvalue of (1.1) is two.

Proof. To prove our assertion it is sufficient to prove that $\lambda_{1}(1)<\lambda_{2}(0)$. Since $\lambda_{2}(0)$ is the second eigenvalue of the eigenvalue problem

$$
v^{\prime \prime}(\phi)+\cot \phi v^{\prime}(\phi)+\lambda v(\phi)=0, v\left(\phi_{1}\right)=v\left(\phi_{2}\right)=0,
$$

by the classical Sturm-Liouville theory, $v_{2}(\phi ; 0)$ have only one nodal point in $\left(\phi_{1}, \phi_{2}\right)$. Let $\phi_{3}$ be the nodal point of $v_{2}(\phi ; 0)$. Then there exist $\xi, \eta$ such that $\phi_{1}<\xi<\phi_{3}<$ $\eta<\phi_{2}$, and $v_{2}{ }^{\prime}(\xi ; 0)=v_{2}{ }^{\prime}(\eta ; 0)=0$. Denote $f(\phi)=v_{2}{ }^{\prime}(\phi ; 0)$. If we differentiate the equation in (2.3) with respect to $\phi$, we find that

$$
f^{\prime \prime}(\phi)+\cot \phi f^{\prime}(\phi)+\left(\lambda_{2}(0)-\frac{1}{\sin ^{2} \phi}\right) f(\phi)=0, f(\xi)=f(\eta)=0
$$

i.e., $\lambda_{2}(0)$ is an eigenvalue of the problem

$$
f^{\prime \prime}(\phi)+\cot \phi f^{\prime}(\phi)+\left(\lambda-\frac{1}{\sin ^{2} \phi}\right) f(\phi)=0, f(\xi)=f(\eta)=0 .
$$

Note that $\lambda_{1}(1)$ is the first eigenvalue of the problem

$$
v^{\prime \prime}(x)+\cot \phi v^{\prime}(\phi)+\left(\lambda-\frac{1}{\sin ^{2} \phi}\right) v(\phi)=0, v\left(\phi_{1}\right)=v\left(\phi_{2}\right)=0 .
$$

Since $(\xi, \eta)$ is a proper subinterval of $\left(\phi_{1}, \phi_{2}\right)$, the first eigenvalue of (2.5) is strictly less than that of $(2.4)$. Hence $\lambda_{1}(1)<\lambda_{2}(0)$. This completes the proof. 
Following Theorem 2.1 we find that the nodal set of a second eigenfunction of a spherical band consists of two longitudes which separate the spherical band into two equal parts.

\section{The extremal Problem for $\lambda_{2}(\mathcal{B})$}

In this section, we prove the following theorem

Theorem 3.1. Let $0<A<1$, and let $\mathcal{B}_{0}$ be the spherical band symmetric with respect to the equator and with area $2 \pi A$. Then, for all bands $\mathcal{B}$ with given area $2 \pi A$ we have

$$
\lambda_{2}(\mathcal{B}) \leq \lambda_{2}\left(\mathcal{B}_{0}\right)
$$

Before proving the theorem, we need some preliminary observations and results. Note that $\lambda_{2}(\mathcal{B})$ coincides with the first eigenvalue of the problem

$$
\frac{d}{d s}\left\{\left(4 s-s^{2}\right) f^{\prime}(s)\right\}+\left(\nu-\frac{4}{4 s-s^{2}}\right) f(s)=0, f\left(s_{1}\right)=f\left(s_{2}\right)=0
$$

which is obtained from (2.5) via change of variables

$$
s=2(1-\cos \phi), \quad s_{1}=2\left(1-\cos \phi_{1}\right), \quad s_{2}=2\left(1-\cos \phi_{2}\right) .
$$

If $A<2$ is fixed and $2 \pi\left(s_{2}-s_{1}\right)=2 \pi A$ is the area of the band, then the last problem is equivalent to

$$
\left\{\begin{array}{l}
\frac{d}{d s}\left\{\left(4\left(s+s_{1}\right)-\left(s+s_{1}\right)^{2}\right) f^{\prime}(s)\right\}+\left(\nu-\frac{4}{4\left(s+s_{1}\right)-\left(s+s_{1}\right)^{2}}\right) f(s)=0 \\
f(0)=f(2 A)=0
\end{array}\right.
$$

simply by replacing $s$ with $s_{1}+s$.

Let $\nu_{1}\left(s_{1}\right)=\lambda_{2}(\mathcal{B})$, which is the first eigenvalue of the above problem. Let $\mathbb{H}_{0}=W_{0}^{1,2}(0,2 A)$, and define for $f \in \mathbb{H}_{0}$

$$
\mathfrak{R}\left(s_{1} ; f\right)=\int_{0}^{2 A}\left(4\left(s+s_{1}\right)-\left(s+s_{1}\right)^{2}\right)\left(f^{\prime}(s)\right)^{2} d s+\int_{0}^{2 A} \frac{4 f^{2}(s)}{4\left(s+s_{1}\right)-\left(s+s_{1}\right)^{2}} d s .
$$

By the classical Rayleigh-Ritz variational principle (see [1])

$$
\nu_{1}\left(s_{1}\right)=\inf _{f \in \mathbb{H}_{0}} \frac{\mathfrak{R}\left(s_{1} ; f\right)}{\int_{0}^{2 A} f^{2}(s) d s} .
$$

Lemma 3.1. Given $0<A<1$, then for all $s_{1} \geq 0$ with $2 A+s_{1}<2$, if $f$ is a $C^{2}$-function defined in $[0,2 A]$, which is symmetric with respect to $s=A$ and such that $f(0)=f(2 A)=0$ and $f(x)$ is not identically zero for all $x \in(0,2 A)$, we have

$$
\frac{\int_{0}^{2 A}\left(4-2\left(s+s_{1}\right)\right)\left(f^{\prime}(s)\right)^{2} d x}{\int_{0}^{2 A} \frac{\left(1-\frac{2\left(s+s_{1}\right)}{4}\right) f^{2}(s)}{\left(s+s_{1}\right)^{2}\left(1-\frac{s+s_{1}}{4}\right)^{2}} d s}>4 \frac{\int_{0}^{A}\left(f^{\prime}(s)\right)^{2} d s}{\int_{0}^{A} \frac{f^{2}(s)}{\left(s+s_{1}\right)^{2}\left(1-\frac{s+s_{1}}{4}\right)^{2}} d s} .
$$

Proof. Since $\left(f^{\prime}(s)\right)^{2}$ is symmetric with respect to $s=A$ on $[0,2 A]$, we have

$$
\begin{aligned}
& \int_{0}^{2 A}\left(4-2\left(s+s_{1}\right)\right)\left(f^{\prime}(s)\right)^{2} d s \\
= & \int_{0}^{A}\left(4-2\left(s+s_{1}\right)\right)\left(f^{\prime}(s)\right)^{2} d s+\int_{0}^{A}\left(4-2\left(2 A-s+s_{1}\right)\right)\left(f^{\prime}(s)\right)^{2} d s \\
= & 4\left[2-\left(A+s_{1}\right)\right] \int_{0}^{A}\left(f^{\prime}(s)\right)^{2} d s .
\end{aligned}
$$


On the other hand, for $0<s<A, 2 A+s_{1}<2$, we have

$$
\left(s+s_{1}\right)\left(1-\frac{s+s_{1}}{4}\right)=1-\frac{\left(s+s_{1}-2\right)^{2}}{4}<1-\frac{\left(2 A-s+s_{1}-2\right)^{2}}{4} .
$$

Thus if we denote

$$
\mathcal{G}_{s_{1}}(s)=\left(s+s_{1}\right)\left(1-\frac{s+s_{1}}{4}\right),
$$

then we have, for $0<s<A$,

$$
\mathcal{G}_{s_{1}}(s)<\mathcal{G}_{s_{1}}(2 A-s),
$$

where $s_{1}$ satisfies the condition $2 A<2 A+s_{1}<2$. Now if $f(s)$ is an even function in $[0,2 A]$, using (3.7) we have

$$
\begin{aligned}
& \int_{0}^{2 A} \frac{\left(1-\frac{2\left(s+s_{1}\right)}{4}\right) f^{2}(s)}{\left(s+s_{1}\right)^{2}\left(1-\frac{s+s_{1}}{4}\right)^{2}} d s \\
= & \int_{0}^{A} \frac{\left(1-\frac{2\left(s+s_{1}\right)}{4}\right) f^{2}(s)}{\left(s+s_{1}\right)^{2}\left(1-\frac{s+s_{1}}{4}\right)^{2}} d s+\int_{0}^{A} \frac{\left(1-\frac{2\left(2 A-s+s_{1}\right)}{4}\right) f^{2}(s)}{\left(2 A-s+s_{1}\right)^{2}\left(1-\frac{2 A-s+s_{1}}{4}\right)^{2}} d s \\
< & \int_{0}^{A} \frac{\left[\left(1-\frac{2\left(s+s_{1}\right)}{4}\right)+\left(1-\frac{2\left(2 A-s+s_{1}\right)}{4}\right)\right] f^{2}(s)}{\left(s+s_{1}\right)^{2}\left(1-\frac{s+s_{1}}{4}\right)^{2}} d s \\
= & \int_{0}^{A} \frac{\left(\left[2-\left(A+s_{1}\right)\right] f^{2}(s)\right.}{\left(s+s_{1}\right)^{2}\left(1-\frac{s+s_{1}}{4}\right)^{2}} d s .
\end{aligned}
$$

(3.4) follows immediately from (3.5) and (3.8).

Lemma 3.2. Given $0<A<1$, and $s_{1} \geq 0$ with $0<2 A+s_{1}<2$, let $f(x) \in C^{2}[0, A]$ with $f(0)=f^{\prime}(A)=0$ and $f(x)$ is not identically zero. Then

$$
\int_{0}^{A}\left(f^{\prime}(s)\right)^{2} d s \geq \frac{1}{4} \int_{0}^{A} \frac{f^{2}(s)}{\left(s+s_{1}\right)^{2}\left(1-\frac{s+s_{1}}{4}\right)^{2}} d s .
$$

"=" could possibly hold only when $s_{1}=0$.

Proof. At first we treat only the case for $s_{1}>0$. For the case $s_{1}=0$, inequality follows simply from the $s_{1}>0$ inequality by letting $s_{1} \rightarrow 0$. Let $\eta_{1}$ denote the first eigenvalue of the problem

$$
w^{\prime \prime}(s)+\eta \frac{1}{s^{2}\left(1-\frac{s}{4}\right)^{2}} w(s)=0, w\left(s_{1}\right)=w^{\prime}\left(s_{1}+A\right)=0 .
$$

To prove our assertion for $s_{1}>0$ it is sufficient to prove that $\eta_{1}>1 / 4$. If the following transformations are made

$$
\begin{aligned}
& z=\frac{1}{K} \int_{s_{1}}^{s}\left(\frac{1}{s}+\frac{1}{4-s}\right) d s, u=\left(\frac{1}{s}+\frac{1}{4-s}\right)^{1 / 2} w, \\
& \rho=K^{2} \eta, K=\frac{1}{\pi} \int_{s_{1}}^{s_{1}+A}\left(\frac{1}{s}+\frac{1}{4-s}\right) d s,
\end{aligned}
$$

(3.10) can be transformed into

$$
u^{\prime \prime}(z)+[\rho-q(z)] u(z)=0, u(0)=u^{\prime}(\pi)=0,
$$

where $q(z)=\theta^{\prime \prime}(z) / \theta(z)$, and $\theta(z)=\left(\frac{1}{s}+\frac{1}{4-s}\right)^{1 / 2}$. By a direct calculation we obtain $q(z)=\frac{K^{2}}{4}$, so that the first eigenvalue of (3.11) is $\rho_{1}=1 / 4+K^{2} / 4$. Hence we have $\eta_{1}=\frac{1}{4}+\frac{1}{4 K^{2}}>1 / 4$, since $\eta_{1}=\rho_{1} / K^{2}$. 
Remark. Inequality (3.9) belongs to a class of general Wirtinger-type inequalities studied in [2]. In fact, if we take $p(x)=\eta_{1} /\left[s^{2}\left(1-\frac{s}{4}\right)^{2}\right]$, then the equation

$$
y^{\prime \prime}(x)+p(x) y(x)=0, \quad s_{1} \leq x \leq s_{1}+A
$$

possesses a positive solution $y_{1}(x)$ with $y_{1}\left(s_{1}\right)=0, y_{1}^{\prime}\left(s_{1}\right)>0$, since $\eta_{1}$ is the first eigenvalue of (3.10) and the corresponding eigenfunction never vanishes in $\left(s_{1}, s_{1}+A\right)$. Inequality (3.9) follows from Theorem 1.1 in [2].

Now, let us go into the proof of our main theorem.

Proof of Theorem 3.1. It is enough to show that the first eigenvalue $\nu_{1}\left(s_{1}\right)$ of (3.2) attains its maximum at $s_{1}=2-A$. Let $\left(\nu\left(s_{1}\right), f_{1}\left(s_{1} ; s\right)\right)$ denote the first normalized eigenpair of (3.2) with $f_{1}\left(s_{1} ; s\right)>0$ in $(0,2 A)$. Taking $f(s) \in \mathbb{H}_{0}$, we then have

$$
\begin{aligned}
\frac{\partial \Re\left(s_{1} ; f\right)}{\partial s_{1}} & =\int_{0}^{2 A}\left[4-2\left(s+s_{1}\right)\right]\left(f^{\prime}(s)\right)^{2} d s-\int_{0}^{2 A} \frac{\left(1-\frac{s+s_{1}}{2}\right) f^{2}(s)}{\left(s+s_{1}\right)^{2}\left(1-\frac{s+s_{1}}{4}\right)^{2}} d s \\
& =\int_{0}^{2 A}\left[4-2\left(s+s_{1}\right)\right]\left(f^{\prime}(s)\right)^{2} d s-\int_{0}^{2 A} \frac{f^{2}(s)}{\left(s+s_{1}\right)^{2}} d s \\
& +\int_{0}^{2 A} \frac{f^{2}(s)}{\left[4-\left(s+s_{1}\right)\right]^{2}} d s,
\end{aligned}
$$

$$
\frac{\partial^{2} \Re\left(s_{1} ; f\right)}{\partial s_{1}^{2}}=-2 \int_{0}^{2 A}\left(f^{\prime}(s)\right)^{2} d s+\int_{0}^{2 A} \frac{2 f^{2}(s)}{\left(s+s_{1}\right)^{3}} d s+\int_{0}^{2 A} \frac{2 f^{2}(s)}{\left[4-\left(s+s_{1}\right)\right]^{3}} d s,
$$

and

$$
\frac{\partial^{3} \Re\left(s_{1} ; f\right)}{\partial s_{1}^{3}}=-6 \int_{0}^{2 A} \frac{f^{2}(s)}{\left(s+s_{1}\right)^{4}} d s+6 \int_{0}^{2 A} \frac{f^{2}(s)}{\left[\left(4-\left(s+s_{1}\right)\right]^{4}\right.} d s .
$$

Notice that for the case $s_{1}=2-A$ in (3.2), the corresponding spherical band is symmetric with respect to the equator, hence the corresponding first eigenfunction $f_{1}(2-A ; s)$ is symmetric with respect to $s=A$. Hence

$$
\frac{\partial^{3} \Re\left(s_{1} ; f_{1}(2-A ; s)\right)}{\partial s_{1}^{3}}=-6 \int_{0}^{2 A} \frac{f_{1}^{2}(2-A ; s)}{\left(s+s_{1}\right)^{4}} d s+6 \int_{0}^{2 A} \frac{f_{1}^{2}(2-A ; s)}{\left[\left(s+\left(4-2 A-s_{1}\right)\right]^{4}\right.} d s .
$$

Since $0<s_{1}<2-A$, we have $s_{1}<4-2 A-s_{1}$, which implies that

$$
\frac{1}{\left(s+s_{1}\right)^{4}}>\frac{1}{\left[s+\left(4-2 A-s_{1}\right)\right]^{4}}
$$

for all $s$ in $[0,2 A]$. Hence for $0<s_{1}<2-A$,

$$
\frac{\partial^{3} \Re}{\partial s_{1}^{3}}\left(s_{1} ; f_{1}(2-A ; s)\right)<0 .
$$

Also notice that, as

$$
\frac{\partial \Re}{\partial s_{1}}\left(2-A, f_{1}(2-A ; s)\right)=\int_{0}^{2 A}(2 A-2 s)\left(f_{1}^{\prime}(2-A ; s)\right)^{2} d s,
$$

$\left(f_{1}^{\prime}(2-A ; s)\right)^{2}$ is symmetric with respect to $s=A$ on $[0,2 A]$ and $2 A-2 s$ is antisymmetric with respect $s=A$ on $[0,2 A]$, we have

$$
\frac{\partial \Re}{\partial s_{1}}\left(2-A, f_{1}(2-A ; s)\right)=0 .
$$


Moreover, by Lemmas 3.1 and 3.2 and (3.15), we have that

$$
\frac{\partial \Re}{\partial s_{1}}\left(s_{1}, f_{1}(2-A ; s)\right)>0, \text { for } 0<s_{1}<2-2 A .
$$

Hence, we have that $\frac{\partial \Re\left(s_{1} ; f_{1}(2-A ; s)\right)}{\partial s_{1}}$ is concave, $\frac{\partial \Re\left(0^{+} ; f_{1}(2-A ; s)\right)}{\partial s_{1}} \geq 0$ and $\frac{\partial \Re\left(2-A ; f_{1}(2-A ; s)\right)}{\partial s_{1}}=0$, so

$$
\frac{\partial \Re}{\partial s_{1}}\left(s_{1}, f_{1}(2-A ; s)\right)>0, \text { for } 0<s_{1}<2-A .
$$

Therefore, for $0 \leq s_{1}<2-A$,

$$
\nu_{1}(2-A)=\mathfrak{R}\left(2-A, f_{1}(2-A ; s)\right) \geq \mathfrak{R}\left(s_{1}, f_{1}(2-A ; s)\right)>\nu_{1}\left(s_{1}\right) .
$$

This completes the proof.

We believe that Lemmas 3.1 and 3.2, and hence Theorem 3.1] can be extended to the case $0<A<2$. We also conjecture that $\lambda_{2}$ is monotonic in $s_{1}$. We will prove the monotonicity of $\lambda_{2}$ for the flat 2-dimensional case in the following section.

\section{Monotonicity of the Second Dirichlet eigenvalue ON A FLAT 2-DIMENSIONAL ANNULUS OF GIVEN AREA}

Let $A_{1}$ and $A_{2}$ be two fixed positive constants, $A_{1}<A_{2}$ and $\mathcal{A}\left(A_{1}, A_{2}\right)$ denote the annulus $2 A_{1} \leq x^{2}+y^{2} \leq 2 A_{2}$ in $\mathbb{R}^{2}$. We denote $2 \pi A$ as the area of $\mathcal{A}\left(A_{1}, A_{2}\right)$ and $\left\{\lambda_{n}\left(A_{1}\right)\right\}$ as the eigenvalues of the problem

$$
\Delta u+\lambda u=0 \text { on } \mathcal{A}\left(A_{1}, A_{2}\right),\left.\quad u\right|_{\partial \mathcal{A}\left(A_{1}, A_{2}\right)}=0,
$$

which is arranged in ascending order. Then by the same arguments as that in Theorem 2.1] we can show that the second eigenvalue of (4.1) is equal to the first eigenvalue $\nu_{1}$ of the problem

$$
z^{\prime \prime}(r)+\frac{1}{r} z^{\prime}(r)+\left(\nu-\frac{1}{r^{2}}\right) z(r)=0, z\left(\gamma_{1}\right)=z\left(\gamma_{2}\right)=0,
$$

where $\gamma_{j}=\left(2 A_{j}\right)^{1 / 2}$. Following the theory of Bessel functions (see [3, ch. 9] or [6]), we see that $\lambda_{2}\left(A_{1}\right)=\nu_{1}$ is the first zero of

$$
\mathcal{F}(\lambda)=Y_{1}\left(\sqrt{\lambda} \gamma_{1}\right) J_{1}\left(\sqrt{\lambda} \gamma_{2}\right)-Y_{1}\left(\sqrt{\lambda} \gamma_{2}\right) J_{1}\left(\sqrt{\lambda} \gamma_{1}\right),
$$

where $J_{1}(x)$ and $Y_{1}(x)$ denote the first and second kinds of Bessel functions of order 1 , respectively. So if we know how the zeros of $\mathcal{F}(\lambda)$ varies as $A_{1}$ changes, we can know how $\lambda_{2}\left(A_{1}\right)$ changes as $A_{1}$ tends to infinity. However, the investigation for the zeros of $\mathcal{F}(\lambda)$ is a little bit complicated, so we avoid this approach. Note that (4.2) can be transformed into

$$
\frac{d}{d s}\left(4 s w^{\prime}(s)\right)+\left(\nu-\frac{1}{s}\right) w(s)=0, w\left(s_{1}\right)=w\left(s_{2}\right)=0
$$

via the change of variables

$$
s=r^{2}, \quad s_{j}=\gamma_{j}^{2}=2 A_{j}, j=1,2 .
$$

For convenience, we simply replace $s$ with $s_{1}+s$ to get

$$
\frac{d}{d s}\left(4\left(s_{1}+s\right) w^{\prime}(s)\right)+\left(\nu-\frac{1}{s_{1}+s}\right) w(s)=0, w(0)=w(2 A)=0 .
$$


Then we have

Theorem 4.1. Given a positive constant $A>0$, let $0<A_{1}<A_{2}$ be two positive numbers with $\mathcal{A}\left(A_{1}, A_{2}\right)=2 \pi A$, where $\mathbb{A}\left(A_{1}, A_{2}\right)$ is as that defined in section 2 . Denote $\lambda_{2}\left(A_{1}\right)$ to be the second eigenvalue of

$$
\left\{\begin{array}{l}
\Delta u+\lambda u=0 \text { on } \mathbb{A}\left(A_{1}, A_{2}\right) \\
\left.u\right|_{\partial \mathbb{A}\left(A_{1}, A_{2}\right)}=0
\end{array}\right.
$$

Then, as a function of $A_{1}, \lambda_{2}\left(A_{1}\right)$ is monotonically increasing. Moreover

$$
\lim _{A_{1} \rightarrow \infty} \lambda_{2}\left(A_{1}\right)=\infty
$$

Proof. Let $2 A_{j}=s_{j}, j=1,2$, and let $w(s)$ be the normalized eigenfunction corresponding to the first eigenvalue $\nu_{1}\left(s_{1}\right)=\lambda_{2}\left(A_{1}\right)$ of (4.4). Then

$$
\left.\nu_{(} s_{1}\right)=\int_{0}^{2 A} 4\left(s+s_{1}\right)\left[w^{\prime}(s)\right]^{2} d s+\int_{0}^{2 A} \frac{[w(s)]^{2}}{s+s_{1}} d s .
$$

Define the functional

$$
\mathcal{K}\left(s_{1} ; w\right)=\int_{0}^{2 A} 4\left(s+s_{1}\right)\left[w^{\prime}(s)\right]^{2} d s+\int_{0}^{2 A} \frac{[w(s)]^{2}}{s+s_{1}} d s .
$$

Then by the following inequality due to Hardy and Littlewood (4], Thm. 253)

$$
\int_{0}^{\infty} 4\left(f^{\prime}(x)\right)^{2} d x>\int_{0}^{\infty} \frac{f^{2}(x)}{x^{2}} d x
$$

for all $f$ with $f^{\prime} \in L^{2}$ and $f(0)=0$, we have

$$
\frac{\partial \mathcal{K}\left(s_{1} ; w\right)}{\partial s_{1}}=\int_{0}^{2 A} 4\left[w^{\prime}(s)\right]^{2} d s-\int_{0}^{2 A} \frac{w(s)^{2}}{\left(s+s_{1}\right)^{2}} d s>0 .
$$

Hence, for $\tilde{s}<s_{1}$,

$$
\nu_{1}(\tilde{s})<\mathcal{K}(\tilde{s} ; w)<\mathcal{K}\left(s_{1} ; w\right)=\nu_{1}\left(s_{1}\right),
$$

i.e., $\lambda_{2}\left(A_{1}\right)=\nu_{1}\left(s_{1}\right)$ is monotonically increasing.

Next, let us compute the limit of $\lambda_{2}\left(A_{1}\right)$. For $s_{1}$ very large and $f \in W_{0}^{1,2}(0,2 A)$ with $\int_{0}^{2 A} f^{2} d s=1$, we have

$$
4 s_{1} \int_{0}^{2 A}\left[w^{\prime}(s)\right]^{2} d s \leq \int_{0}^{2 A} 4\left(s+s_{1}\right)\left[w^{\prime}(s)\right]^{2} d s+\int_{0}^{2 A} \frac{[w(s)]^{2}}{s+s_{1}} d s .
$$

Taking the infimum at both sides, we have $\lim _{A_{1} \rightarrow \infty} \lambda_{2}\left(A_{1}\right)=\infty$.

\section{ACKnowledgments}

This research was partially supported by the National Science Council, Republic of China. The author thanks the referee of this paper for his helpful suggestions and comments. 


\section{REFERENCES}

1. C. Bandle, Isoperimetric Inequalities and Applications, Pitman, Landon, 1980. MR 81e:35095

2. P. R. Beesack, Integral Inequalities of the Wirtinger Type, Duke. Math. J. vol. 25(1958), pp. 477-498. MR 20:3947

3. R. Courant and D. Hilbert, Methods of Mathematical Physics, vol. I, Wiley-Interscience, New York-London, 1953. MR 16:426a

4. G. H. Hardy, J. E. Littlewood and G. Polya. Inequalities, Cambridge University Press, 1952. MR 13:727e

5. E. L. Ince, Ordinary Differential Equations, Dover, New York, 1956.

6. J. R. Kuttler and V. G. Sigillito, Eigenvalues of the Laplacian in Two Dimensions, SIAM Review, vol 26(1984), no. 2 pp. 163-193. MR 85k:65086

7. C.-L. Shen and C.-T. Shieh, Some properties of the first eigenvalue of the Laplace operator on the spherical bands in $S^{2}$, SIAM J. Math. Anal., vol. 23(1992), 1305-1308. MR 93f:35162

8. G. P. Tolstov, Fourier Series, Dover, New York, 1962.

Department of Mathematics, Fu-Jen Catholic University, Taipei, Taiwan, Republic OF CHINA

E-mail address: ctshieh@math.fju.edu.tw 